\title{
An Expert System to Assess Fire Safety in Dwellings
}

\author{
H. A. DONEGAN, I. R. TAYLOR and R. T. MEEHAN \\ Institute of Informatics \\ University of Ulster \\ Shore Road, Newtownabbey BT37 OOB, UK
}

\section{ABSTRACT}

This paper describes the application of an expert system to the evaluation of fire safety in dwellings based on the body of knowledge developed by the Fire Engineering Research Group at the University of ulster. The background and philosophy of the evaluation procedure together with the associated reasoning with respect to the choice of system and its implementation are outlined in some detail. This demonstration system is intended as a pilot study for a more ambitious programme. A discussion relating to problems with the system and future developments concludes the paper.

KEYWORDS: Fire safety evaluation, expert system, Delphi, points soheme.

\section{INTRODUCTION}

In recent years the notion of fire safety evaluation with respect to buildings has become inextricably bound up with the perception of the prioritisation of those entities which taken together comprise the fire safety components of a specific building type. The prioritisations, often but not exclusively the result of expert opinion, are used in the development of points schemes. These are essentially research into practice devices which facilitate the economical allocation of scarce resources in the design and refurbishment of buildings. The philosophy is clearly extendible to any form of shelter, e.g. ships, planes and trains, where life safety and property protection are paramount. This paper will focus on dwelling fire safety with specific reference to the determination of a fire safety quality measure which can be optimised interactively using the expert system shell. Xi Plus in a PC environment. The level of optimisation achieved is a function of the user's requirements and resources given the existence of acceptable norms.

The chronology of events leading to this work began with the study by Nelson and Shibe [1] who produced a system for the Fire safety Evaluation 
of health care facilities in the USA. Following these developments, the Department of Health and social services (DHSS) sponsored the Fire Engineering Department at the University of Edinburgh to produce a fire safety evaluation points scheme for patient areas within hospitals. The work was conducted by Marchant [2] and completed in 1982. Stollard [3] reported on the development of the points scheme at Edinburgh and outlined the procedures which were used. In 1983 a pilot study [4] based on the methodology of Marchant was undertaken to consider the application of the DHSS scheme to dwellings. This led to a programme of work in 1984 at the University of Ulster, funded by the Science and Engineering Research Council. The theoretical consequences [5] and practical considerations [6] which followed provide the immediate environment for the present expert system.

The motivation for the expert system derives from the complexity of the total. fire safety scheme and the need to create a 'what if' environment for the user. The ideas in [2] and [4] are directed towards the production of a weighted ranking, referred to as a priority vector, of fire safety components. Typical components might be management, occupants and visitors, contents, fire brigade, detection systems, fire fighting equipment and so on. The weighted rankings for $n$ such components $\left(w_{1}, w_{2}, w_{3}, \ldots w_{n}\right)$ emerge from the consensus of expert opinion generally

obtained through an application of the Delphi process [7]. Notwithstanding certain shortcomings of the latter [8] and assuming that a priority vector can be arrived at, the fundamental and as yet unanswered question is not with regard to its existence, but with regard to its significance in the field of application. More particularly its optimal effectiveness - can it be used to minimize safety maintenance costs or to allocate scare resources in the pursuit of maximum safety? The authors would contend that such issues in the environment of fire safety analysis are the driving force behind research into practice. On the premise that total safety while being a desirable objective is something which can only be approximated within technological development periods, the approximation is presumably the stability of professional expert opinion within each time period.

Fire safety history provides a wealth of examples of connected time periods, each new period arising as a result of at least one catastrophe at the conclusion of or within a previous time period. The overall effect has been the production of more and more prescriptive legislation which inhibits an engineering approach to the solution of many problems. At the present time, within the constraints of existing legislation, consideration is being given to engineering strategies which will facilitate a degree of trade-off between passive and active fire safety measures. This is where the application of an expert system capable of logical repeatability has a distinct advantage in that it provides a basis for uniformity in the decision making process. It is clear from Figure 1 that an expert system which provides the user with a 'what if' environment fits comfortably as part of a generalisation of the simple fire safety framework proposed in [5].

Prior to the introduction of the expert system the procedure in using a points scheme involved taking the scalar product of the priority vector $v^{\prime}=\left(w_{1}, w_{2}, w_{3}, \ldots w_{n}\right)$ with each of two corresponding sets of component 


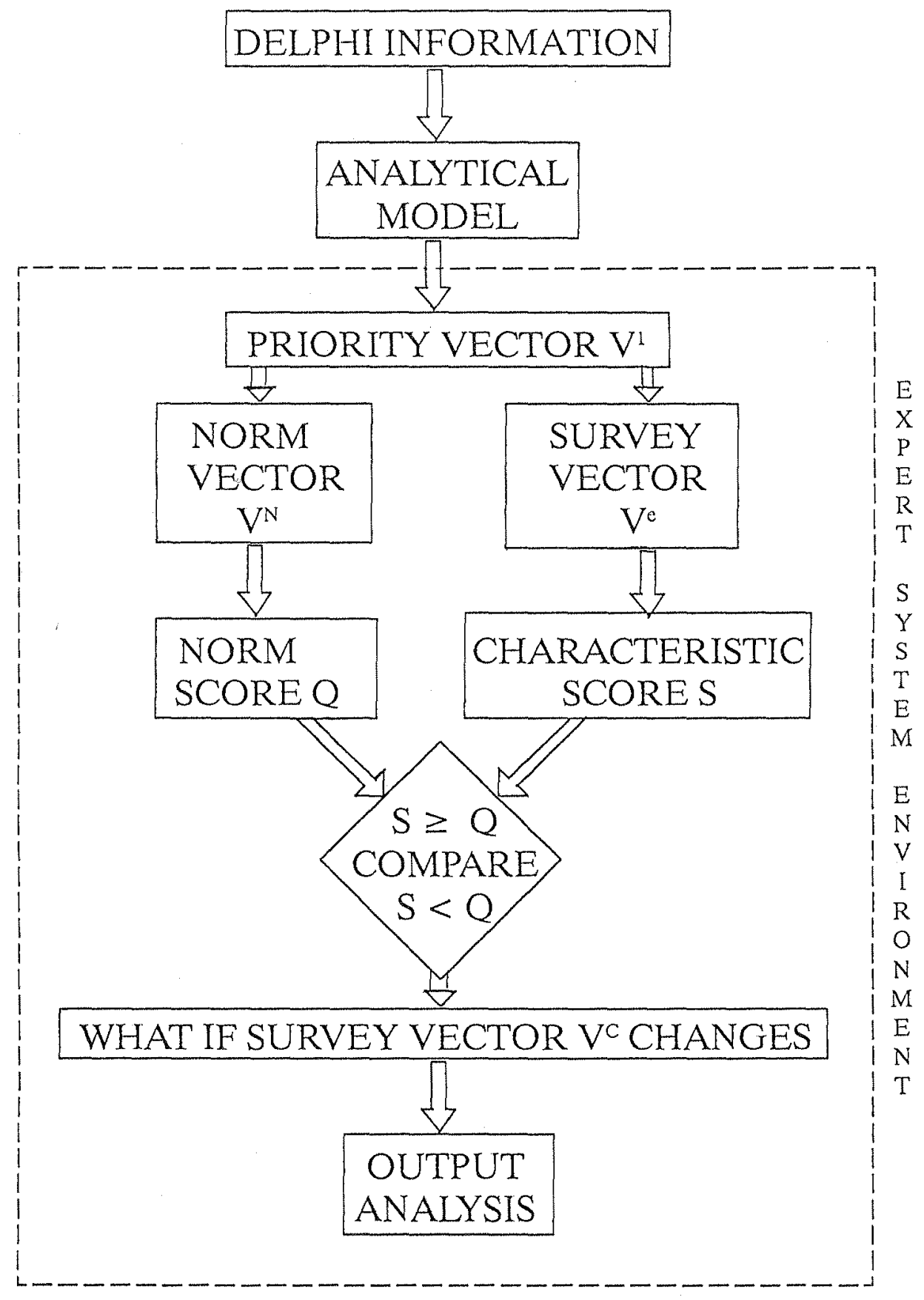

MIG. 1 GLNERALISIND FRAMIWORK FOR FIRU SAFETY EVAI,UATION 
points score allocations - a norm set $v^{N}=\left(q_{1}, q_{2}, q_{3}, \ldots q_{n}\right)$ for a specific dwelling category and a survey set $v^{C}=\left(s_{1}, s_{2}, s_{3}, \ldots s_{n}\right)$ for an actual dwelling in the category [4]. The corresponding products, the norm score $Q$ and characteristic score $s$ were then compared.

The advantage of the above generalisation became apparent when it was realised that it was quite feasible for a dwelling to satisfy the overall criterion, viz: $S>Q$ and yet fail in one or more subsets of the components. Given that there are $2^{n}-1$ mathematically possible subsets for the $n$ components this can lead to a large number of possibilities for large $n$. However in this case with $n=11$, [6], it was decided to cluster the components from a fire engineering point of view as follows:

A: Human measures

B: Building specific or passive measures and

C: Supportive or active measures.

Table 1 exemplifies the categorisation used in this study.

TABLE 1. Fire Safety Component Clustering

\begin{tabular}{|l|l|l|}
\hline A:Human & B:Passive & C:Active \\
\hline Occupants and & Internal Design & Eire Brigade \\
Visitors & Survey Volume & Detection Systems \\
Contents & Means of Escape & First Aid Fire \\
Management & Hazard Protection & Fighting Equipment \\
& External Envelope & \\
\hline
\end{tabular}

An important feature of the conceptualisation was to distinguish the notions of passive/active safety from passive/active measures. Literature on safety [9] defines active safety as accident prevention and passive safety as accident protection, with the proviso that every critical event is a series of casual events within which it is possible to practice accident prevention (active safety). Once the critical event occurs it is only possible to practice accident protection (passive safety). Given the situation of a critical event (an unwanted fire), fire engineering literature refers to active and passive measures designed to combat the event. It is clear that trade-off between active and passive measures is possible but there is no potential for trade-off between active and passive safety. Figure 2 shows the fundamental relationship between these variables.

From this diagram it is possible to see that total fire safety will only arise as a result of complete active safety or total prevention whereas within a technological time period passive safety is the limiting state of prevailing expert opinion. The implicit assumption is that as technological time periods progress there should be a statistically observable decrease in fatalities and in property destruction. The efficiency of any system can only be measured in the future. Meanwhile the present approach is a contribution to the organised thinking which is essential for any level of improved safety. The next sections will describe the choice of application and its implementation. 


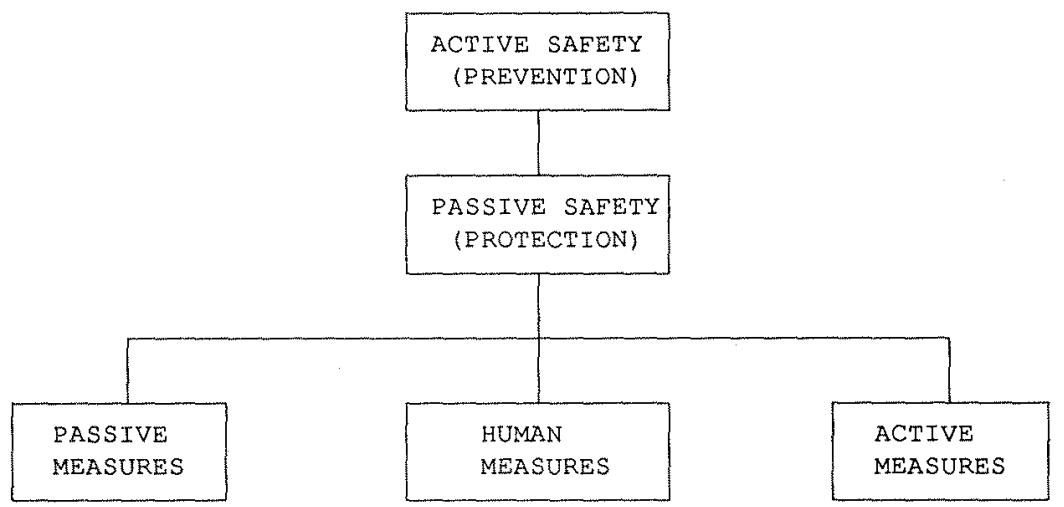

FIGURE 2 Passive/Active Safety and Passive/Active Measures

\section{CHOICE OF SYSTEM}

Bearing in mind the above considerations the resultant expert system must be capable of:

1 - deciding overall if a dwelling is adequate for fire safety,

2 - allowing some trade off between active and passive measures,

3 - suggesting acceptable requirements on each of the measures $A, B$ and $C$ and of

4 - allowing changes in the dwelling specification to test 'what if' questions.

It was decided to investigate if an expert system could meet the above requirements with the possibility that such a system could also answer "why" questions, justifying its conclusions. Such a computer system should also be consistent in its answers and allow a variety of people in different locations to access its expertise. By building in help information at various levels, the system can be employed by more users, allowing them to learn from the inbuilt expertise. To aid such use, information about the dwelling under consideration must be input, with the system asking appropriate questions and using default values if no information is available.

To test its feasibility the authors have applied the expert system to the study of the previousiy mentioned points system for evaluating fire safety in dwellings, $[4,6,10]$. The eleven components of fire safety, as identified by the Delphi technique, are clustered into the $A, B$ and $C$ categories described above. The expertise of the system includes identification of the eleven components, their relative weighting as given by the vector $V^{\prime}$ and the norm score $Q$ for which a particular kind of dwelling would be deemed to satisfy fire safety requirements. An expert system requires a knowledge base within which such expertise is represented, an inference engine to process the knowledge and an interface to users and developers. Within an expert system shell the latter two facilities are already provided allowing the user to concentrate on the development of the knowledge base. Since the shell is 'domain-free' and so contains no information on fire safety, the knowledge base must contain all 
the expertise of the system, which as well as rules and facts includes appropriate questions and help for the user. For this investigation the $P C$ based shell $X i$ Plus was chosen, in which the knowledge is represented in a rule-based form. The shell allows links to, for example, databases, spreadsheets and $C$ programs and this was utilised for some of the data. The shell provides a comprehensive set of tools for developing and testing the knowledge base, within a user friendly environment. While it does not allow for probabilities within the data this was not required in the present project.

Relatively little work on the application of expert systems within fire safety has been published, with most of it on the compliance of buildings with fire regulations. The best known example is the commercial program BRIGADE [11], a system with 4000 rules based on the shell Level 5 , while there are discussions of ongoing work in [12].

\section{IMPLEMENTATION}

In developing an expert system the main problems are obtaining the expertise and then deciding how to represent it within the knowledge base. In basing this expert system on the work of $[4,6]$, the acquisition of knowledge using the Delphi technique has been performed and it remains to represent it within the shell. The components of Table 1 are structured as illustrated as in figure 3, with appropriate questions, on-line help and corresponding rules for each component.

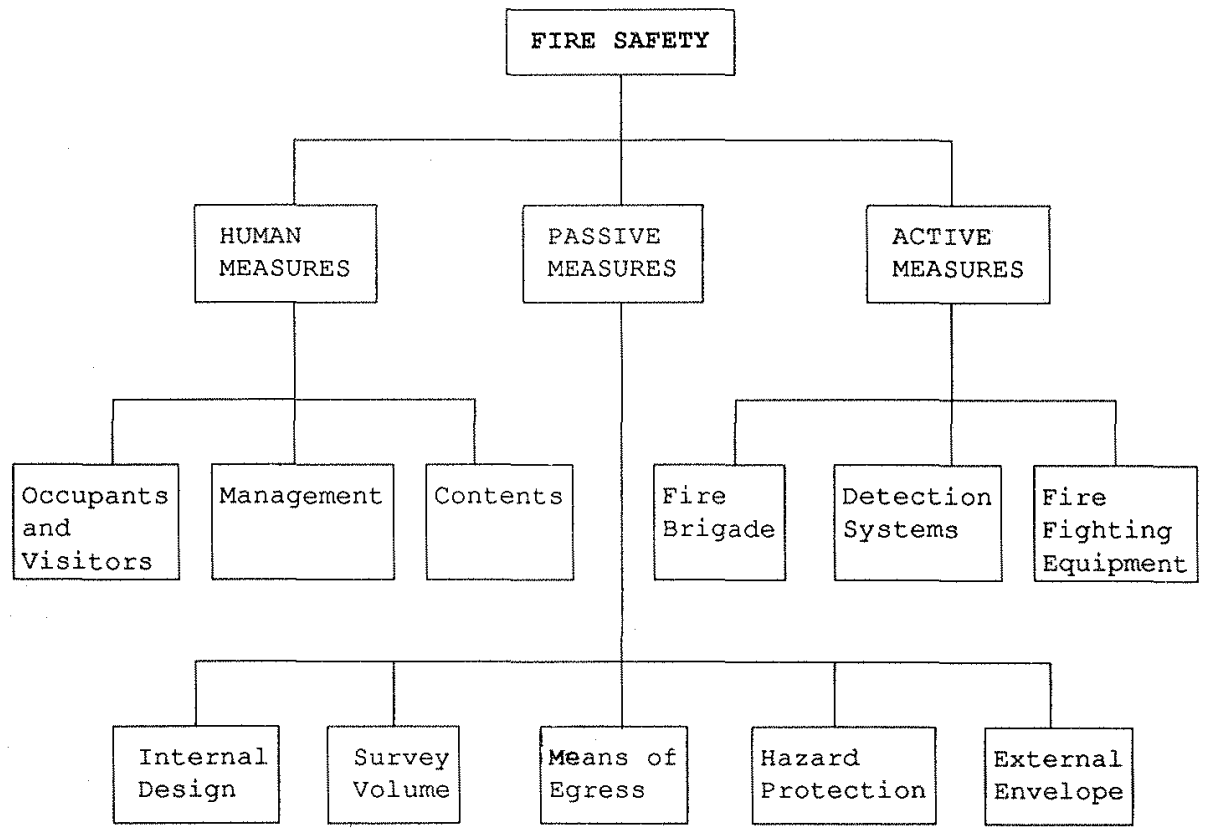

FIGURE 3 External Knowledge structure 
In the system as described in [6], worksheets were used to assess each of the eleven components with a rating on a scale from 0-5. Each component set was then weighted by the priority vector $V^{\prime}$ to give an overall score, $S$ in a scale 0-500. Expert opinion indicates that for the house type considered in the investigation an overall score of 375 or $75 \%$ would be sufficient for the dwelling to satisfy fire safety standards. This equates with norm scores for each measure of $(A, 154),(B, 154)$ and $(C, 67)$, each being $75 \%$ of the corresponding maximum allocations of $(A, 205),(B, 205)$ and $(C, 90)$. To allow for trade-off between the different measures the selected norm scores for $A, B$ and $C$ must be less than $75 \%$ of the overall maximum allocation. This is illustrated in the Results section below.

Within the expert system, separate knowledge bases were constructed for the three measures $A, B$ and $C$, aiding separate development and testing. The information required is obtained by prompting the user with various questions. The answers are tested by the inbuilt rules of the knowledge base and along with other information stored as data the program decides on an overall score for the dweling.

Unlike the manual system, the expert system can allow different levels of trade-off between A, B and C by setting separate norms for these measures. For example in obtaining a rating for "occupants and visitors" the information considered is the number of occupants compared with the number of bedspaces, the number of floors and the ratio of adults to dependents. A screen of the form of figure 4 is used to request information, with help available if required. Within this component there are rules such as

IF number of occupants = bed spaces

THEN occupant score $=1.7$

If dwelling is two storey

THEN survey score $=1.3$

with a total of 17 rules for the first component. Under $A$ for human measures there are 46 rules, in $B$ on building specific measures there are 80 rules with 34 rules in $C$. To test a system with a total of 160 rules, as many as possible paths through the system must be tried. A set of test cases representing standard and extreme situations was developed, to probe the system for potential limitations and weaknesses.

\section{RESULTS}

Applying the program to an actual dwelling, typical output might be

A: Human Measures

B: Building Specific Measures

C: Supportive Measures OVERALI

$\begin{array}{ccc}\text { actual } & \text { selected } & \\ \text { score } & \text { norm score } & \text { pass/fail } \\ 170 & 141 & \mathrm{p} \\ 165 & 135 & \mathrm{p} \\ 50 & 58 & \mathrm{f} \\ 385 & 375 & \mathrm{p}\end{array}$


Application: Fire Safety

Knowledgebase: Human Measures

\begin{tabular}{|l|l|}
\hline How many occupants in dwelling? & 3 \\
How many bed spaces in dwelling? & 2 \\
What size is dwelling? & $\begin{array}{l}\text { single storey } \\
\text { *two storey } \\
\text { greater than two storey }\end{array}$ \\
How many able adults in dwelling? & 1 \\
How many dependants in dwelling? & 2 \\
PRESS F1 FOR HELP
\end{tabular}

Tab/Backtab next/previous field

Esc cancel || CTRL+Rtn end || E3 why \|

FIGURE 4: Information requested on occupants and visitors, within human measures.

To allow trade-off the selected norm scores in this example are (A, 141), $(B, 135)$ and $(C, 58)$. The overall required score is of course 375 (75\% of 500). Trade-off is occuring between $C$, below standard, and $A$ and $B$ to give an overall pass for the dwelling.

In contrast for another dwelling the results may be

$\begin{array}{lccc} & \text { actual } & \text { selected } & \\ \text { score } & \text { norm score } & \text { pass/fail. } \\ \text { A: Human Measures } & 145 & 141 & \mathrm{p} \\ \text { B: Building Specific Measures } & 140 & 135 & \mathrm{p} \\ \text { C: Supportive Measures } & 70 & 58 & \mathrm{p} \\ \text { OVERALI } & 355 & 375 & \mathrm{f}\end{array}$

so that the dwelling fails to reach the required standard even though it passes in each of $A, B$ and $C$. If trade-off is allowed, by setting the norms for $A, B$ and $C$ below the overall standard required, then inevitably some dwellings will pass each aspect but fail to reach the required overall standard, as in this example. By setting the required norm scores for $A, B$ and $C$ at 154,154 and 67 respectively, ie $75 \%$ of each maximum possible score of 205,205 and 90, no trade-off would be allowed between the measures. The philosophy of trade-off is discussed further in [13].

If a dwelling fails to reach the overall standard, the expert system allows a 'what if' type investigation to be performed to ascertain what improvements may be made to bring the house up to specification. With the adaition of some financial information the alternate cost of a range of possible alterations to the building may be explored using the expert system. The program can be easily adjusted to allow trade-off only between the passive and active measures, $B$ and $C$. 


\section{CONCLUSIONS}

This paper shows that a points scheme for the fire safety evaluation of dwellings may be computerised using an expert system. The program illustrates the merits of using an expert system shell to encapsulate the knowledge, especially in comparison to using a conventional programming language. The program can be run at all stages of development with a built-in user friendliness. On any consultation, context sensitive help is available to the user at the level required, the user may ask the system why some information is required and explore 'what if' type situations. When loaded in a portable PC, the system could be employed on-site to assess the status of a dwelling and, if required, to suggest options for improving its level of fire safety to any required level. The program allows trade-off between different aspects of fire safety, but the amount of trade-off may be adjusted. The system could be developed to assess the fire safety of for example public assembly buildings as discussed in [14]. on the basis of this pilot study the authors are convinced that subject to appropriate funding, the expert system incorporating a points scheme could be extended to more complex buildings where the risk factors are likely to be much higher.

\section{REFERENCES}

1. Nelson, H.E., and Shibe, A.J., "A system of Fire Safety Evaluation of Health Care Facilities", Report NBSIR 78-1555-1, NBS, 1978.

2. Marchant, E.W., (ed), "Fire Safety Evaluation (Points) Scheme for Patient Areas within Hospitals", A report on its origins and development sponsored by DHSS Department of Fire Safety Engineering, University of Edinburgh, 1982.

3. Stollard, P., "The Development of a Points Scheme to Assess Fire Safety in Hospitals", Eire Safety Journal, 7, 145-153, 1984.

4. Shields, T.J. Silcock, G.W. and Bell Y., "Fire Safety Evaluation of Dwellings", Fire Safety Journal, 10, 29-36, 1986.

5. Donegan, H.A., Shields T.J., and Silcock G.W., "A Mathematical Strategy to Relate Fire Safety Evaluation and Fire Safety Policy Formulation for Buildings" in Fire Safety Science - Proceedings of the 2nd International Symposium, Tokyo, pp. 433-441, 1988.

6. Shields, T.J, Silcock G.W. and Donegan, H.A., "Assessing Fire Risk in Dwellings", University of Uister, 1989.

7. Linstone, H.A. and Turoff, M., (eds), The Delphi Method, Techniques and Applications, Addison Wesley, 1975 .

8. Shields, T.J., Silcock, G.W. and Donegan, H.A., "Methodological Problems Associated with the Use of the Delphi Technique", Fire Technology, 23, 175-186, 1987.

9. Wilson, G.A., "Techniques of Safety and Risk Management", Proc. One Day Seminar, Dept. of Mech. and Ind. Eng., University of Ulster and Plant Safety, 22, 2, 1990.

10. Shields, T.J., Silcock, G.W. and Donegan, H.A., "The Development of a Fire Safety Evaluation Points Scheme for Dwellings, Part I - Some Theoretical Considerations", Fire Safety Journal, 15, 313-324, 1989.

11. Peregrine Expert Systems Ltd, "Brigade", Dublin, 1988 .

12. Hamilton G., Harrison A.P., Pascall J.R., Directory of Research and development of expert systems in the construction and building 
services industries Vol III, BSRIA Ref 7177, 1983.

13. Shields T.J., Silcock G.W. and Donegan H.A., "A Philosophy for TradeOff", Report for Department of Environment, NI, 1989.

14. Shields, T.J., Silcock G.W. and Donegan, H.A.," Towards the

development of a fire safety systems evaluation for public assembly buildings", Construction Management and Economics, 8, 147-158, 1990. 